\title{
Relation of the nonlinear Heisenberg algebras in two dimensions with linear ones
}

\author{
Won Sang Chung ${ }^{1}$
}

Received: 22 December 2014/ Accepted: 8 June 2015/Published online: 17 July 2015

(C) The Author(s) 2015. This article is published with open access at Springerlink.com

\begin{abstract}
In this paper, we discuss the relation of the nonlinear Heisenberg algebras in two dimensions with linear ones following the Nowicki and Tkachuk's approach for one-dimensional case. For one-dimensional harmonic oscillator, we obtain the solution explicitly. For the nonlinear Heisenberg algebras in two dimensions, we introduce two generators to transform this algebra into the linear one. For the linear version of the nonlinear Heisenberg algebras in two dimensions, we obtain the eigenfunction for the position and angular momentum operator and solve the harmonic oscillator problem in two dimensions.
\end{abstract}

Keywords Nonlinear Heisenberg algebras · Algebra . Two dimensions

\section{Introduction}

The first form of the Heisenberg algebra giving the minimal length uncertainty was first introduced by Kempf, Mangano, and Mann [1] in the following form:

$$
[X, P]=i \hbar\left(1+\beta P^{2}\right),
$$

which suggests the existence of the fundamental minimal length

$$
(\Delta X)_{0}=\hbar \sqrt{\beta} .
$$

In this direction, much development has been accomplished in order to study the effect of minimal length on the

Won Sang Chung

mimip4444@hanmail.net

1 Department of Physics and Research Institute of Natural Science, College of Natural Science, Gyeongsang National University, Jinju 660-701, Korea quantum physical systems as well as on the classical ones, but only a few problems are shown to be solved exactly. They are one-dimensional harmonic oscillator with minimal length uncertainty in position $[1,2]$ and also with minimal length uncertainty in position and momentum [3, 4], D-dimensional isotropic harmonic oscillator [5, 6], three-dimensional Dirac oscillator [7], $(1+1)$-dimensional Dirac oscillator within Lorentz-covariant deformed algebra [8], one-dimensional Coulomb problem [9], and the singular inverse square potential with a minimal length $[10,11]$. Three-dimensional Coulomb problem with deformed Heisenberg algebra was solved within the perturbation theory [12-15].

In this paper, we discuss the relation of the nonlinear Heisenberg algebras in two dimensions with linear ones following the Nowicki and Tkachuk's approach [16] for one-dimensional case. For one-dimensional harmonic oscillator, we obtain the solution explicitly. For the nonlinear Heisenberg algebras in two dimensions, we introduce two generators to transform this algebra into the linear one. For the linear version of the nonlinear Heisenberg algebras in two dimensions, we obtain the eigenfunction for the position and angular momentum operator and solve the harmonic oscillator problem in two dimensions.

\section{One-dimensional deformed nonlinear Heisenberg algebra}

Recently, Nowicki and Tkachuk [16] considered a onedimensional deformed nonlinear Heisenberg algebra with function of deformation $f(P)$, namely

$$
[X, P]=\text { if }(P) \text {, }
$$

where $f(P)$ is an positive function obeying 
$f(-P)=f(P)$.

It means that the space has the same properties in two opposite directions. The momentum representation reads

$P=p, \quad X=i f(p) \partial_{p}$

and acts on the square integrable functions $\phi(p) \in$ $\mathcal{L}^{2}(-a, a ; f),(a \leq \infty)$ where the norm of $\phi$ is given by

$\|\phi\|^{2}=\int_{-a}^{a} \frac{\mathrm{dp}}{f(p)}|\phi(p)|^{2}$.

More general momentum representation is given in "Appendix".

Nowicki and Tkachuk extended the algebra (3) into the three generator algebra by one additional generator $F=$ $f(p)$ and obtain a concrete form of $f$ as follows:

$f(p)=\sqrt{1+\beta p^{2}}$.

Now let us consider the harmonic oscillator with the hamiltonian

$H=\frac{1}{2 \mu} P^{2}+\frac{1}{2} \mu w^{2} X^{2}$.

The Schrödinger equation reads

$$
\left[\frac{p^{2}}{2 \mu}+\frac{1}{2} \mu w^{2}\left(i \sqrt{1+\beta p^{2}} \partial_{p}\right)^{2}\right] \psi=E \psi \text {. }
$$

Let us change the variable like $\sqrt{\beta} p=\sinh \xi$, which means that $\xi$ goes to zero when $\beta$ approaches zero. Then, Eq. (9) becomes

$\psi^{\prime \prime}+\left(\epsilon-\frac{\sinh ^{2} \xi}{(\beta \mu w)^{2}}\right) \psi=0$,

where

$\epsilon=\frac{2 E}{\beta \mu w^{2}}$.

When we consider the small value of $\xi$, we have

$\psi^{\prime \prime}+\left(\epsilon-\sigma \xi^{2}\right) \psi=0$,

where

$$
\sigma=\frac{1}{(\beta \mu w)^{2}} .
$$

Replacing $\xi^{2}=z$, we get

$4 z \psi^{\prime \prime}+2 \psi^{\prime}+(\epsilon-\sigma z) \psi=0$.

If we set

$\psi(z)=e^{-\frac{z}{2 \mu w}} y(z)$,

we get $z y^{\prime \prime}+\left(\frac{1}{2}-\frac{1}{\mu w} z\right) y^{\prime}+\frac{1}{4}\left(\epsilon-\frac{1}{\mu w}\right) y=0$.

This equation can be solved using the Frobenius method. If we adopt

$y(z)=\sum_{n=0}^{\infty} a_{n} z^{n+\lambda}$

and insert it into Eq. (15), we have

$$
\begin{aligned}
& a_{0} \lambda(\lambda-1 / 2)=0 \\
& (n+1+\lambda)(n+1 / 2+\lambda) a_{n+1} \\
& \quad=\left[-\frac{1}{4}\left(\epsilon-\frac{1}{\mu w}\right)+\frac{1}{\mu w}(n+\lambda)\right] a_{n}, \quad(n=0,1, \ldots) .
\end{aligned}
$$

From the characteristic Eq. (17), we have two values of $\lambda$ : $\lambda=0,1 / 2$.

For $\lambda=0$, we have

$$
y_{1}(z)={ }_{1} F_{1}\left(\frac{1}{4}(1-\mu w \epsilon) ; \frac{1}{2} ; \frac{1}{\mu w}\right)
$$

and for $\lambda=1 / 2$, we have

$y_{2}(z)={ }_{1} F_{1}\left(\frac{1}{4}(3-\mu w \epsilon) ; \frac{3}{2} ; \frac{1}{\mu w}\right)$,

where Kummer's function is defined as

$$
{ }_{1} F_{1}(a ; b ; z)=\sum_{n=0}^{\infty} \frac{(a)_{n} z^{n}}{n !(b)_{n}}
$$

and

$$
(a)_{0}=1, \quad(a)_{n}=a(a+1)(a+2) \cdots(a+n-1),
$$

is the rising factorial.

\section{Linearization of a two-dimensional deformed nonlinear Heisenberg algebra}

Let us consider a two-dimensional deformed nonlinear Heisenberg algebra with deformation function $f(P)$ :

$$
\left[X_{1}, P_{1}\right]=\text { if }(P), \quad\left[X_{2}, P_{2}\right]=\text { if }(P), \quad\left[P_{1}, P_{2}\right]=0,
$$

where $f(P)=f\left(P_{1}, P_{2}\right)$ is an positive function obeying

$f\left(-P_{1}, P_{2}\right)=f\left(P_{1}, P_{2}\right), \quad f\left(P_{1},-P_{2}\right)=f\left(P_{1}, P_{2}\right)$.

It means that the space has the same properties in two opposite $X_{1}$ - and $X_{2}$-directions. From the commutation 
relations (23), we have the momentum representation of the operators as follows:

$$
\begin{aligned}
& P_{1}=p_{1}, \quad P_{2}=p_{2} \\
& X_{1}=i f(p) \partial_{p_{1}}, \quad X_{2}=i f(p) \partial_{p_{2}} .
\end{aligned}
$$

This fixes the remaining commutation relations, so the full algebra is then given by

$$
\begin{aligned}
& {\left[X_{1}, P_{1}\right]=i f(P), \quad\left[X_{2}, P_{2}\right]=i f(P), \quad\left[P_{1}, P_{2}\right]=0} \\
& {\left[X_{1}, X_{2}\right]=-f\left(\partial_{P_{1}} f \partial_{P_{2}}-\partial_{P_{2}} f \partial_{P_{1}}\right), \quad\left[X_{1}, P_{2}\right]=\left[X_{2}, P_{1}\right]=0 .}
\end{aligned}
$$

Indeed one can easily check that the relation (26) obeys the Jacobi identity.

Now we assume that the operators $X_{1}, X_{2}, P_{1}$ and $P_{2}$ act on the square integrable functions $\phi\left(p_{1}, p_{2}\right) \in$ $\mathcal{L}^{2}(-a, a ; f),(a \leq \infty)$ where the norm of $\phi$ is given by

$\|\phi\|^{2}=\int_{-a}^{a} \int_{-a}^{a} \frac{\mathrm{d} p_{1} \mathrm{~d} p_{2}}{f(p)}|\phi(p)|^{2}$.

For the self-adjointness of $X_{1}$ and $X_{2}$, we have

$\phi\left(-a, p_{2}\right)= \pm \phi\left(a, p_{2}\right)$

and

$\phi\left(p_{1},-a\right)= \pm \phi\left(p_{1}, a\right)$.

Now we extend this algebra by two additional operators

$F=f(P)$

$G=i f\left(\partial_{P_{1}} f \partial_{P_{2}}-\partial_{P_{2}} f \partial_{P_{1}}\right)$.

Thus, the extended algebra $\mathcal{E}$ is generated by the six generators. Using representation (25), one can easily find

$\left[X_{1}, F\right]=$ if $\partial_{P_{1}} f, \quad\left[X_{2}, F\right]=$ if $\partial_{P_{2}} f, \quad\left[P_{1}, F\right]=\left[P_{2}, F\right]=0$.

We require that both $\left\{X_{1}, P_{1}, F\right\}$ and $\left\{X_{2}, P_{2}, F\right\}$ form a subalgebras of $\mathcal{E}$. Then, one can put

$$
\begin{aligned}
& f \partial_{P_{1}} f=\alpha+\beta P_{1}+\gamma F \\
& f \partial_{P_{2}} f=\alpha^{\prime}+\beta^{\prime} P_{2}+\gamma^{\prime} F,
\end{aligned}
$$

where $\alpha, \beta, \gamma, \alpha^{\prime}, \beta^{\prime}, \gamma^{\prime}$ are real parameters. Note that the linear combination in the right-hand side of (30) (or 31) does not contain $X_{1}$ (or $X_{2}$ ) because $f \partial_{P_{1}} f$ (or $f \partial_{P_{2}} f$ ) is a function of $P_{1}, P_{2}$ only. Using Eq. (24) and changing $P_{1}, P_{2}$ into $-P_{1},-P_{2}$, respectively, one find

$f \partial_{P_{1}} f=-\alpha+\beta P_{1}-\gamma F$ $f \partial_{P_{2}} f=-\alpha^{\prime}+\beta^{\prime} P_{2}-\gamma^{\prime} F$

Comparing Eqs. (30) (31) or with Eqs. (32) (or 33), one can see

$f \partial_{P_{1}} f=\beta P_{1}, \quad f \partial_{P_{2}} f=\beta^{\prime} P_{2}$.

From now on we will restrict our concern to the case of $\beta=\beta^{\prime}=v^{2}>0$ for simplicity. From Eq. (34), we have

$f(P)=\sqrt{1+\beta P_{1}^{2}+\beta P_{2}^{2}}$.

Thus, the algebra $\mathcal{E}$ reads

$$
\begin{aligned}
& {\left[X_{1}, X_{2}\right]=i G, \quad\left[X_{1}, P_{1}\right]=i F, \quad\left[X_{1}, P_{2}\right]=0} \\
& \quad\left[X_{1}, F\right]=i \beta P_{1}, \quad\left[X_{1}, G\right]=i \beta X_{2}, \\
& {\left[X_{2}, P_{1}\right]=0, \quad\left[X_{2}, P_{2}\right]=i F, \quad\left[X_{2}, F\right]=i \beta P_{2}, \quad\left[X_{2}, G\right]} \\
& \quad=-i \beta X_{1}, \quad\left[P_{1}, P_{2}\right]=0, \\
& {\left[P_{1}, F\right]=0, \quad\left[P_{1}, G\right]=i \beta P_{2}, \quad\left[P_{2}, F\right]=0} \\
& \quad\left[P_{2}, G\right]=-i \beta P_{1}, \quad[F, G]=0 .
\end{aligned}
$$

This is Lie algebra. One can find the Casimir operator (invariant) for this algebra

$$
K=P_{1}^{2}+P_{2}^{2}-\frac{1}{\beta} F^{2}
$$

commuting with all elements of the algebra. Now let us define the following operators:

$$
\begin{array}{ll}
A_{1}=v P_{1}, & A_{2}=F, \quad A_{3}=\frac{1}{v} X_{1} \\
A_{4}=\frac{1}{v} X_{2}, & A_{5}=G, \quad A_{6}=v P_{2} .
\end{array}
$$

Then, the algebra $\mathcal{E}$ can be written as

$$
\begin{aligned}
& {\left[A_{1}, A_{2}\right]=0, \quad\left[A_{3}, A_{1}\right]=i A_{2}, \quad\left[A_{3}, A_{2}\right]=i A_{1}} \\
& {\left[A_{4}, A_{2}\right]=i A_{6}, \quad\left[A_{4}, A_{6}\right]=i A_{2}, \quad\left[A_{6}, A_{2}\right]=0} \\
& {\left[A_{5}, A_{1}\right]=-i v^{2} A_{6}, \quad\left[A_{5}, A_{6}\right]=i v^{2} A_{1}, \quad\left[A_{1}, A_{6}\right]=0} \\
& {\left[A_{3}, A_{4}\right]=i \frac{1}{v^{2}} A_{5}, \quad\left[A_{4}, A_{5}\right]=-i v^{2} A_{3}, \quad\left[A_{5}, A_{3}\right]=-i v^{2} A_{4}} \\
& {\left[A_{1}, A_{4}\right]=0, \quad\left[A_{2}, A_{5}\right]=0, \quad\left[A_{3}, A_{6}\right]=0 .}
\end{aligned}
$$

The algebra $\mathcal{E}$ possess some subalgebras:

1. subalgebra $\mathcal{E}_{1}$ generated by $A_{1}, A_{2}, A_{3}$

2. subalgebra $\mathcal{E}_{2}$ generated by $A_{2}, A_{4}, A_{6}$

3. subalgebra $\mathcal{E}_{3}$ generated by $A_{1}, A_{5}, A_{6}$

4. subalgebra $\mathcal{E}_{4}$ generated by $A_{3}, A_{4}, A_{5}$.

It is convenient to use two pairs of commuting hermitian operators $P_{ \pm}$and $Q_{ \pm}$defined as follows: 
$P_{+}=A_{1}+A_{2}=v P_{1}+F$

$P_{-}=A_{2}-A_{1}=F-v P_{1}$

$Q_{+}=A_{2}+A_{6}=v P_{2}+F$

$Q_{-}=A_{2}-A_{6}=F-v P_{2}$.

Indeed one can easily check that

$$
\left[P_{ \pm}, Q_{ \pm}\right]=0
$$

In this case, we have the algebra $\mathcal{A}$ generated by $P_{ \pm}, Q_{ \pm}, A_{3}, A_{4}, A_{5}$. Algebra $\mathcal{E}$ has six generators, while $\mathcal{A}$ has seven ones. It seems to be nonsense because two algebras should be isomorphic. To cure this problem, let us consider the inverse relations of Eq. (40)

$A_{1}=\frac{1}{2}\left(P_{+}-P_{-}\right), \quad A_{2}=\frac{1}{2}\left(P_{+}+P_{-}\right)$,

$A_{2}=\frac{1}{2}\left(Q_{+}+Q_{-}\right), \quad A_{6}=\frac{1}{2}\left(Q_{+}-Q_{-}\right)$.

We know that $A_{2}$ can be expressed in terms of both $P_{ \pm}$and $Q_{ \pm}$, which gives a constraint

$P_{+}+P_{-}=Q_{+}+Q_{-}$.

This constraint decreases the number of generators of the algebra $\mathcal{A}$, so two algebras are isomorphic.

Besides Eq. (41), the remaining commutation relations of the algebra $\mathcal{A}$ are

$$
\begin{gathered}
{\left[A_{3}, P_{ \pm}\right]= \pm i P_{ \pm}, \quad\left[P_{+}, P_{-}\right]=0} \\
{\left[A_{4}, Q_{ \pm}\right]= \pm i Q_{ \pm}, \quad\left[Q_{+}, Q_{-}\right]=0} \\
{\left[A_{3}, Q_{ \pm}\right]=\frac{i}{2}\left(P_{+}-P_{-}\right), \quad\left[A_{4}, P_{ \pm}\right]=\frac{i}{2}\left(Q_{+}-Q_{-}\right),} \\
{\left[A_{5}, Q_{ \pm}\right]=\frac{i}{2} v^{2}\left(P_{+}-P_{-}\right), \quad\left[A_{5}, P_{ \pm}\right]=\mp i v^{2} A_{6}} \\
{\left[A_{3}, A_{4}\right]=\frac{i}{v^{2}} A_{5}, \quad\left[A_{4}, A_{5}\right]=i v^{2} A_{3}, \quad\left[A_{5}, A_{3}\right]=-i v^{2} A_{4},}
\end{gathered}
$$

The Casimir operator is then given by

$K=\frac{1}{v^{2}}\left(A_{2}^{2}-P_{+} P_{-}-Q_{+} Q_{-}\right)$.

This algebra has two subalgebras (namely $I S O(1,1)$ ) generated by $A_{3}, P_{ \pm}$and $A_{4}, Q_{ \pm}$.

Two sets of the ladder operators can be expressed in terms of the momentum operators as follows:

$$
\begin{aligned}
& P_{ \pm}=\sqrt{1+v^{2}\left(P_{1}^{2}+P_{2}^{2}\right)} \pm v P_{1}, \\
& Q_{ \pm}=\sqrt{1+v^{2}\left(P_{1}^{2}+P_{2}^{2}\right)} \pm v P_{2} .
\end{aligned}
$$

If we set $v P_{1}=\sinh \xi \cos \eta, \quad v P_{2}=\sinh \xi \sin \eta$,

we can express $P_{ \pm}$and $Q_{ \pm}$as

$P_{ \pm}=\cosh \xi \pm \sinh \xi \cos \eta$

$Q_{ \pm}=\cosh \xi \pm \sinh \xi \sin v \eta$.

The $\xi$ and $\eta$ can be expressed in terms of the momentum operators as

$\eta=\tan ^{-1}\left(\frac{P_{2}}{P_{1}}\right), \quad \xi=\sinh ^{-1} \sqrt{\left(v P_{1}\right)^{2}+\left(v P_{2}\right)^{2}}$.

Then, $A_{3}, A_{4}, A_{5}$ can be written as

$A_{3}=i\left[\cos \eta \partial_{\xi}-\operatorname{coth} \xi \sin \eta \partial_{\eta}\right]$

$A_{4}=i\left[\sin \eta \partial_{\xi}+\operatorname{coth} \xi \cos \eta \partial_{\eta}\right]$

$A_{5}=\frac{i}{v} \partial_{\eta}$

\section{Eigenvectors of the position operator and angular momentum operator}

In this section, we discuss the eigenvalue equation for the position operator and angular momentum operator. The eigenvalues for the position operators read

$X_{1} \phi(\xi, \eta)=l_{1} w(\xi, \eta) \phi(\xi, \eta)$

$X_{2} \phi(\xi, \eta)=l_{2} w(\xi, \eta) \phi(\xi, \eta)$,

where $w(\xi, \eta)$ is a weight function. Inserting Eq. (50) into Eq. (51) yields

$$
\begin{aligned}
& i v\left[\cos \eta \partial_{\xi}-\operatorname{coth} \xi \sin \eta \partial_{\eta}\right] \phi(\xi, \eta)=l_{1} w(\xi, \eta) \phi(\xi, \eta) \\
& i v\left[\sin \eta \partial_{\xi}+\operatorname{coth} \xi \cos \eta \partial_{\eta}\right] \phi(\xi, \eta)=l_{2} w(\xi, \eta) \phi(\xi, \eta) .
\end{aligned}
$$

From Eq. (52), we have

$\partial_{\xi} \phi=\frac{w}{i v}\left(l_{1} \cos \eta+l_{2} \sin \eta\right) \phi$

$\operatorname{coth} \xi \partial_{\eta} \phi=\frac{w}{i v}\left(l_{2} \cos \eta-l_{1} \sin \eta\right) \phi$

If we set

$\phi(\xi, \eta)=X(\xi) Y(\eta), \quad w(\xi, \eta)=w_{1}(\xi) w_{2}(\eta)$,

we have

$$
\begin{aligned}
X^{\prime} & =\frac{1}{i v} w_{1}(\xi) w_{2}(\eta)\left(l_{1} \cos \eta+l_{2} \sin \eta\right) X \\
Y^{\prime} & =\frac{1}{i v} w_{1}(\xi) w_{2}(\eta)\left(l_{2} \cos \eta-l_{1} \sin \eta\right) Y .
\end{aligned}
$$

If we adopt 
$w_{1}=-i \operatorname{coth} \xi, \quad w_{2}=\frac{1}{l_{1} \cos \eta+l_{2} \sin \eta}$,

we have

$\phi(\xi, \eta)=\left[\sinh \xi\left(l_{1} \cos \eta+l_{2} \sin \eta\right)\right]^{-1 / v}$.

Now let us investigate the eigenvalue of the angular momentum operator $L$ defined as

$L=\epsilon_{i j} X_{i} P_{j}=i\left(f P_{2} \partial_{P_{1}}-f P_{1} \partial_{P_{2}}\right)=-i \cosh \xi \partial_{\eta}$.

The eigenvalue equation reads

$L \Phi_{m}=m \hbar \Phi_{m}, \quad(m \in Z)$.

Solving Eq. (58), we have

$\Phi_{m}(\xi, \eta)=\frac{1}{\cosh \xi} e^{i m \hbar \eta}$

\section{Two-dimensional Harmonic oscillator}

Now let us consider the isotropic harmonic Hamiltonian

$\hat{H}=\frac{1}{2 \mu}\left(P_{1}^{2}+P_{2}^{2}\right)+\frac{1}{2} \mu w^{2}\left(X_{1}^{2}+X_{2}^{2}\right)$.

Using Eq. (47) and Eq. (50), we obtain the expression of $H$ :

$\hat{H}=-\frac{\mu w^{2} v^{2}}{2}\left(\partial_{\xi}^{2}+\operatorname{coth} \xi \partial_{\xi}+\operatorname{coth}^{2} \xi \partial_{\eta}^{2}\right)+\frac{1}{2 \mu v^{2}} \sinh ^{2} \xi$.

Then, the Schrödinger equation reads

$$
\left[-\frac{\mu w^{2} v^{2}}{2}\left(\partial_{\xi}^{2}+\operatorname{coth} \xi \partial_{\xi}+\operatorname{coth}^{2} \xi \partial_{\eta}^{2}\right)+\frac{1}{2 \mu v^{2}} \sinh ^{2} \xi\right] \psi=E \psi .
$$

If we set $\psi=R(\xi) e^{i m \eta}$, we have

$R^{\prime \prime}+\operatorname{coth} \xi R^{\prime}+\left(\epsilon-A \sinh ^{2} \xi-m^{2} \operatorname{coth}^{2} \xi\right) R=0$,

where

$\epsilon=\frac{2 E}{\mu w^{2} v^{2}}, \quad A=\frac{1}{\mu^{2} w^{2} v^{4}}$.

Now consider the case that $\xi$ is sufficiently small. In this case, we have

$\sinh \xi \approx \xi, \quad \operatorname{coth} \xi \approx \frac{1}{\xi}$.

Then, Eq. (63) reduces to

$R^{\prime \prime}+\frac{1}{\xi} R^{\prime}+\left(\epsilon-A \xi^{2}-\frac{m^{2}}{\xi^{2}}\right) R=0$.

If we set $\xi^{2}=\mu w v^{2} z$, we have $z R^{\prime \prime}+R^{\prime}+\left(\frac{1}{4} \mu w v^{2} \epsilon-\frac{1}{4} z-\frac{m^{2}}{4 z}\right) R=0$.

Solving Eq. (65), we get

$R=e^{-\frac{\xi^{2}}{2 \mu w v^{2}}}\left(\frac{\xi^{2}}{\mu w v^{2}}\right)^{\frac{m+1}{2}} L_{n}^{m}\left(\frac{\xi^{2}}{\mu w v^{2}}\right)$

and

$E_{n m}=\hbar w(2 n+m+1), \quad n=0,1, \ldots, \quad m=0,1, \ldots, n$.

The ground state energy is given by

$E_{00}=\hbar w$

which corresponds to the classical result.

\section{Conclusion}

Recently, Nowicki and Tkachuk [16] considered a onedimensional deformed nonlinear Heisenberg algebra with function of deformation $f(P)$, namely $[X, P]=$ if $(P)$. They discussed the relation of the nonlinear Heisenberg algebras with linear ones. We introduced the variable $\xi=$ $\sinh ^{-1}(\sqrt{\beta} p)$ to solve the one-dimensional harmonic problem for the small value of $\xi$. We extended Nowicki and Tkachuk's work to the two-dimensional case. We obtained the linearized algebra $\mathcal{E}$ from the two-dimensional nonlinear Heisenberg algebras by adding two generators. Introducing two variables

$$
\begin{aligned}
& \xi=\sinh ^{-1}\left(v^{2} p_{1}^{2}+v^{2} p_{2}^{2}\right) \\
& \eta=\tan ^{-1}\left(\frac{p_{2}}{p_{1}}\right)
\end{aligned}
$$

we expressed all generators of the algebra $\mathcal{E}$ in terms of $\xi$ and $\eta$. We also solved the eigenvalue equation for the position and angular momentum operator. Finally, we discussed twodimensional isotropic harmonic oscillator problem and obtained the corresponding energy eigenvalue and wave function for the small value of $\xi$. We found that the ground state energy for this model corresponds to the classical result.

Acknowledgments This Work (GNUDFF- 2014-25) was supported by the Academy-oriented Research Funds of Development Fund Foundation, Gyeongsang National University, 2014.

Open Access This article is distributed under the terms of the Creative Commons Attribution 4.0 International License (http://creativecommons.org/licenses/by/4.0/), which permits unrestricted use, distribution, and reproduction in any medium, provided you give appropriate credit to the original author(s) and the source, provide a link to the Creative Commons license, and indicate if changes were made. 


\section{Appendix}

More general solution of Eq. (3) is

$X=i f(p) \partial_{p}+i g(p)$.

We assume that the norm of $\phi$ takes the form

$$
\|\phi\|^{2}=\int_{-a}^{a} \mathrm{~d} p \mu(p)|\phi(p)|^{2}
$$

where the measure function obeys $\mu(-p)=\mu(p)$. For the self-adjointness of $X$, we have

$\mu^{\prime} f+\left(f^{\prime}-2 g\right) \mu=0$.

Solving Eq. (71), we get

$\mu(p)=\frac{1}{f} e^{2 \int \frac{g}{f} d p}$

Ex.1 For $g=0$, we have $\mu=\frac{1}{f}$.

Ex.2 For $f=1+\beta p^{2}, g=\gamma p$, we have $\mu=f^{\gamma / \beta-1}$.

Ex.3 For $f=\sqrt{1+\beta p^{2}}, g=\gamma p$, we have $\mu=\frac{1}{\sqrt{1+\beta p^{2}}} e^{\frac{2 \gamma}{\beta} \sqrt{1+\beta p^{2}}}$.

\section{References}

1. Kempf, A., Mangano, G., Mann, R.B.: Hilbert space representation of the minimal length uncertainty relation. Phys. Rev. D 52, 1108 (1995)

2. Kempf, A.: Noncommutative geometric regularization. Phys. Rev. D 54, 5174 (1996)

3. Quesne, C., Tkachuk, V.M.: Deformed algebras, position-dependent effective masses and curved spaces: an exactly solvable Coulomb problem. J. Phys. A 36, 10373 (2003)
4. Quesne, C., Tkachuk, V.M.: More on a SUSY QM approach to the harmonic oscillator with nonzero minimal uncertainties in position and/or momentum. J. Phys. A 37, 10095 (2004)

5. Chang, L.N., Minic, D., Okamura, N., Takeuchi, T.: Exact solution of the harmonic oscillator in arbitrary dimensions with minimal length uncertainty relations. Phys. Rev. D 65, 125027 (2002)

6. Dadic, I., Jonke, L., Meljanac, S.: Harmonic oscillator with minimal length uncertainty relations and ladder operators. Phys. Rev. D 67, 087701 (2003)

7. Quesne, C., Tkachuk, V.M.: Dirac oscillator with nonzero minimal uncertainty in position. J. Phys. A 38, 1747 (2005)

8. Quesne, C., Tkachuk, V.M.: Lorentz-covariant deformed algebra with minimal length and application to the $(1+1)$-dimensional Dirac oscillator. J. Phys. A 39, 10909 (2006)

9. Fityo, T.V., Vakarchuk, I.O., Tkachuk, V.M.: One-dimensional Coulomb-like problem in deformed space with minimal length. J. Phys. A 39, 2143 (2006)

10. Bouaziz, D., Bawin, M.: Regularization of the singular inverse square potential in quantum mechanics with a minimal length. Phys. Rev. A 76, 032112 (2007)

11. Bouaziz, D., Bawin, M.: Singular inverse square potential in arbitrary dimensions with a minimal length: application to the motion of a dipole in a cosmic string background. Phys. Rev. A 78, 032110 (2008)

12. Brau, F.: Minimal length uncertainty relation and hydrogen atom. J. Phys. A 32, 7691 (1999)

13. Benczik, S., Chang, L.N., Minic, D., Takeuchi, T.: Hydrogenatom spectrum under a minimal-length hypothesis. Phys. Rev. A 72, 012104 (2005)

14. Stetsko, M.M., Tkachuk, V.M.: Perturbation hydrogen-atom spectrum in deformed space with minimal length. Phys. Rev. A 74, 012101 (2006)

15. Stetsko, M.M.: Corrections to the ns levels of the hydrogen atom in deformed space with minimal length. Phys. Rev. A 74, 062105 (2006)

16. Nowicki, A., Tkachuk, V.: Relation of deformed nonlinear algebras with linear ones. J. Phys. A: Math. Theor. 47, 025207 (2014) 